\title{
Feature Tracking by Multi-frame Relaxation
}

\author{
Nigel G Sharp and Edwin R Hancock* \\ Department of Computer Science \\ University of York \\ York, Y01 5DD, UK
}

\begin{abstract}
This paper describes a novel feature tracking method. It is based on an interframe relaxation technique. This method combines intra-frame and inter-frame constraints on the behaviour of acceptable contour structure. The intra-frame information is represented by both a dictionary of local contour structure and a statistical model of the response of a set of directional feature detection operators. The inter-frame ingredient represents the novel modelling component; it is encapsulated by an implicit model of the underlying surface structure of 3D feature points. The model is represented in terms of a series of unimodal probability densities whose single parameter is the interframe distance. The initial probabilities in our relaxation scheme effectively combine distributions describing the statistical uncertainties in the position and feature characteristics of multiframe contours; these probabilities are refined in the light of the dictionary to produce consistent contours. We present an experimental evaluation of the resulting feature detection method on cranial MRI data. Here the method significantly outperforms its single frame counterpart in term of its ability to extract noise-free and smooth feature contours.
\end{abstract}

\section{Introduction}

Effective feature tracking is an essential ingredient in both motion analysis and volumetric image reconstruction. The basic task involves the robust identification and location of feature contours that exhibit interframe deformations caused either by object motion or by significant surface curvature. In order to be effective, tracking must be capable of distinguising genuine variations in both the position, shape and intensity when the available image information is noisy or uncertain. Existing techniques described in the literature can be divided into those that attempt to capture contour deformation at either the global or local levels. Global methods $[1,7,10]$ aim to locate acceptable parametric contour deformations that satisfy constraints on consistent interframe movement [1] and closeness to the raw intensity data [10]. However, their main limitation stems from the need for prior knowledge governing the topology of the target contours [15]. If this knowledge is not to hand, then local tracking methods offer an attractive alternative which also effectively draw on information concerning intensity structure [11] and consistency. However, while

\footnotetext{
*Nigel Sharp is supported by EPSRC.
} 
the global methods accommodate this information in a unified optimisation framework, local feature tracking methods draw upon it sequentially in a multistage approach.

The localised multistage philosophy is nicely exemplified in the volumeteric imaging domain. Here MRI slice analysis is invariably approached as a three stage segmentation strategy $[7,11,12]$. Firstly, intensity features which persist over a significant number of adjacent slices are labelled as potential surface points $[13,14]$. In practice the detected features are invariably $3 \mathrm{D}$ edges which present themselves as intensity discontinuities [11]. As with 2D edges, hysteresis linking is applied to improve the connectivity of the detected intensity features; for instance Monga, Deriche and Malandain [11] have developed an extension of the Canny-Deriche [2,3] edge detector to 3D slice data processing. The next stage involves fitting surfaces to the $3 \mathrm{D}$ feature points and subsequent refinement of the surface parameters to ensure local consistency. Various fitting techniques are available for the surface refinement process. Sander and Zucker [13,14] adopt a conventional least-squares approach while Monga, Ayache and Sander [12] use a Kalman filter in a weighted least squares fit.

We have recently developed a novel relaxation framework [5] which has proved extremely robust when applied to $2 \mathrm{D}$ feature detection problems $[5,6,8,9]$. It has the unique feature of combining uncertain intensity information in the light of constraints on consistent contour structure. This unified framework also holds out the promise of enhanced 3D feature detection capabilities both in terms of its performance and its capacity to represent local surface structure $[5,6,8]$. It has been extensively and successfully applied to the processing of a variety of $2 \mathrm{D}$ intensity features including steps [5] and ridges as represented either by the output of an orientational quadrature filter bank [9] or by a resolution pyramid [8]. Moreover, recent studies reveal that the method can unambiguously resolve the two feature types when quadrature filters are used [9]. Much of its robustness stems from the use of contextual information, in particular a dictionary representation of $2 \mathrm{D}$ contour structures [5], in the refinement of intensity-features whose uncertainties are characterised by probability density functions. These probability densities directly model the effects of applying image operators to the raw image data. In consequence, their parameters may be directly estimated from the raw-image statistics, endowing the relaxation approach with adaptive capabilities [6]. Under certain limiting conditions [6], the relaxation approach may be viewed as an adaptive version of Canny's hysteresis linking idea [2].

Our aim in this paper is to develop a relaxation process for multiframe processing $[11,10]$. The motivation for this study stems from the need to directly exploit interframe persistence contraints in the feature detection process; this is to be contrasted with the multistage approach to volumetric feature detection. Although feature contours on individual frames may be expected to exhibit considerable variation in contrast and relative position, they share the property of being the 2D manifestations of 3D surfaces [14]. For these reasons, we would like to avoid a model that is based on the constancy of either intensity or local spatial proximity. It is therefore the 2D connectivity [5] of contours and their implicit membership of 3D surfaces [14] that we wish to exploit in constructing a model of interframe feature persistence. Our implicit 3D surface model is extremely simple, it is based on the assumption of uniformity of surface orientation and mutual exclusivity of edgel origin. These assumptions lead to a model that has all the hallmarks of a locally deformable template $[1,10,15]$.

The relaxation process is concerned with combining evidence. In so doing it simultaneously accommodates the statistical processes at play in intensity feature characterisation 
via filtering operations $[6,7]$ and in generating interframe contour deformations. Feature characteristics derived from filtering the raw image data are modelled by an additive Gaussian noise model while positional contour movements are attributed to the local geometry of the underlying 3D surface. Moreover, constraints on 2D image structure in the individual slices are accommodated by a local dictionary of consistent contours [5]. This dictionary not only embodies the intra-slice behaviour of contour structure, it also imposes constraints on the interslice evolution of consistent 3D surface traces. The dictionary may be regarded as a set of local surface templates. Relaxation operations are aimed at locating locally consistent contour structure based on the evidential information provided by the intensity characteristics of the features being tracked and by variations in their positions due to underlying surface movements. The feature contours in different slices may therefore be regarded as undergoing implicit deformation in the light of evidence provided by feature characteristics and constraints provided by the dictionary.

The outline of this paper is follows. Section 2 develops the multi-frame relaxation framework necessary to formulate the feature tracking process. Section 3 presents models of $2 \mathrm{D}$ contour structure, interframe feature persistence and statistical feature characterisation. In Section 4 we apply the resulting models to the tracking of 3D features in cranial MRI data. Finally, Section 5 offers some conclusions and suggests directions for future investigation.

\section{Bayesian Framework}

The novelty of the work reported here is to extend the evidence combining ideas of Hancock and Kittler [5] to interframe feature tracking. Our aim is to develop a relaxation framework which is applicable to the tracking of features in multiple image sequences. Although the main experimental vehicle for this study is the reconstruction of $3 \mathrm{D}$ intensity features in an MRI stack [7,11,12], many of the ideas are applicable to other applications involving image sequences such as motion analysis $[1,10]$. In order to commence our formal development of the multi-frame relaxation formula, we must specify the form and nature of the information available to us.

The first element is to specify the information available to us from the different frames. To be more formal, suppose that the image entities forming the frame with index $L$ are denoted by the set $F^{L}$; this set consists of the Cartesian pairs representing the pixel co-ordinates in the frame of interest. We will be interested in labelling the pixels in the frame $F^{L}$ by combining evidence in the form of within-frame measurements together with labelled feature contours from the previous frame in the sequence, i.e. $F^{L-1}$. We let $\underline{x}_{i, j}^{L}$ to denote the measurement-vector pertaining to the pixel $(i, j) \in F^{L}$. In order to curb the complexity of our relaxation scheme we will confine our attention to the measurements contained within the $3 \times 3$ neighbourhood $I_{i, j}^{L}$ of $\operatorname{pixel}(i, j)$. The aim of applying relaxation operations is to label each pixel in the frame $F^{L}$ using the set of available labels, $\Omega$. In our feature detection application we will be interested in labelling contours associated with intensity ridges or ravines. In this example the label-set consists of two directional feature labels $\leftrightarrow$ and $\uparrow$, associated with vertical and horizontal features, together with a null-feature category $\phi$. The labelled representation of the frame consists of the assigned labels at each pixel element; it is denoted by the set $\Upsilon^{L}=\left\{\theta_{i, j}^{L} \in \Omega, \forall(i, j) \in F^{L}\right\}$.

Our aim in performing relaxation operations is to allow interframe information to prevail on the interpretation of pixels at each succeeding frame in a sequence. We therefore commence the labelling of the sequence at the frame indexed zero using a conventional 
relaxation method and propagate consistent labellings down the image stack. Once the relaxation operations on a particular frame have converged upon a consistent hard interpretation, the resulting labelling may be utilised as an additional source of contextual information to track features in the subsequent frames in the sequence. Additional information of this sort can only effectively enhance the quality of interpretation provided that a suitable model of interframe feature persistence is available. It is this model requirement that places the greatest demands on our multi-frame relaxation method.

Meeting the demands outlined above requires significant extensions to the single frame evidence combining framework of Hancock and Kittler [5]. The starting point in the development of a single-frame relaxation formula was to consider the $a$ posteriori probability for the object-label assignments conditioned only upon the measurement information from the contextual neighbourhood. According to this framework, the probability updating scheme was interpreted as an implicit iterative filtering operation on the available measurements. The basis for the multiframe relaxation formula is a simple extension of this basic probability updating scheme. Rather than conditioning of the $a$ posteriori probability upon single-frame observations alone, we also take into consideration the object-label assignments obtained from the previous frame in the sequence. With this additional information source at hand the development of the multiframe relaxation scheme involves consideration of the following $a$ posteriori probability

$$
P\left(\theta_{i, j}^{L} \mid \underline{x}_{k, l}^{L}, \forall(k, l) \in I_{i, j}^{L}, \Upsilon^{L-1}\right)
$$

The development of a detailed evidence combining formula from this new starting point proceeds along the lines adopted in [5] and leads to the following non-linear probability update formula

$$
P^{(n+1)}\left(\theta_{i, j}^{L}\right)=\frac{P^{(n)}\left(\theta_{i, j}^{L}\right) Q^{(n)}\left(\theta_{i, j}^{L}\right)}{\sum_{\theta_{i, j}^{L} \in \Omega} P^{(n)}\left(\theta_{i, j}^{L}\right) Q^{(n)}\left(\theta_{i, j}^{L}\right)}
$$

Evidence for the label assignment $\theta_{i, j}^{L}$ is accumulated over the contextual neighbourhood $I_{i, j}^{L}$ in frame $F^{L}$ by the support function $Q^{(n)}\left(\theta_{i, j}^{L}\right)$. In their studies of single frame relaxation, Kittler and Hancock [5] found the most effective strategy for developing practical relaxation schemes was to derive support functions appropriate to specific object arrangement topologies. One of the more powerful ideas developed in connection with the single frame relaxation approach was that of using a dictionary of legal vocabulary symbol configurations to model the joint prior [5]; only configurations inside the dictionary have a non-zero a priori probability of occurrence. The dictionary for the ensemble with indexset $I_{i, j}^{L}$ is denoted by $\Theta$ and contains $Z$ entries. Let $\lambda_{k, l}^{h}$ denote the label on the object indexed $(k, l)$, corresponding to the $h$ th entry in the dictionary $\Theta$, then we can introduce the following shorthand notation for the dictionary items $\Lambda^{h}=\left\{\lambda_{k, l}^{h}, \forall(k, l) \in I_{i, j}^{L}\right\}$. The dictionary itself is then the set of consistent labellings $\Theta=\left\{\Lambda^{h}, h=1, Z\right\}$. For the $2 \mathrm{D}$ feature detection application the dictionary consists of all the connected contours which can be constructed on a $3 \times 3$ neighbourhood using the label-set $\Omega=\{\leftrightarrow, \uparrow, \phi\}$. There are about 100 such configurations; some typical examples are shown in Figure 1. All the consistent contours are a single pixel wide. The dictionary contains symbolic representations of important feature points such as corners and T-junctions. With the 

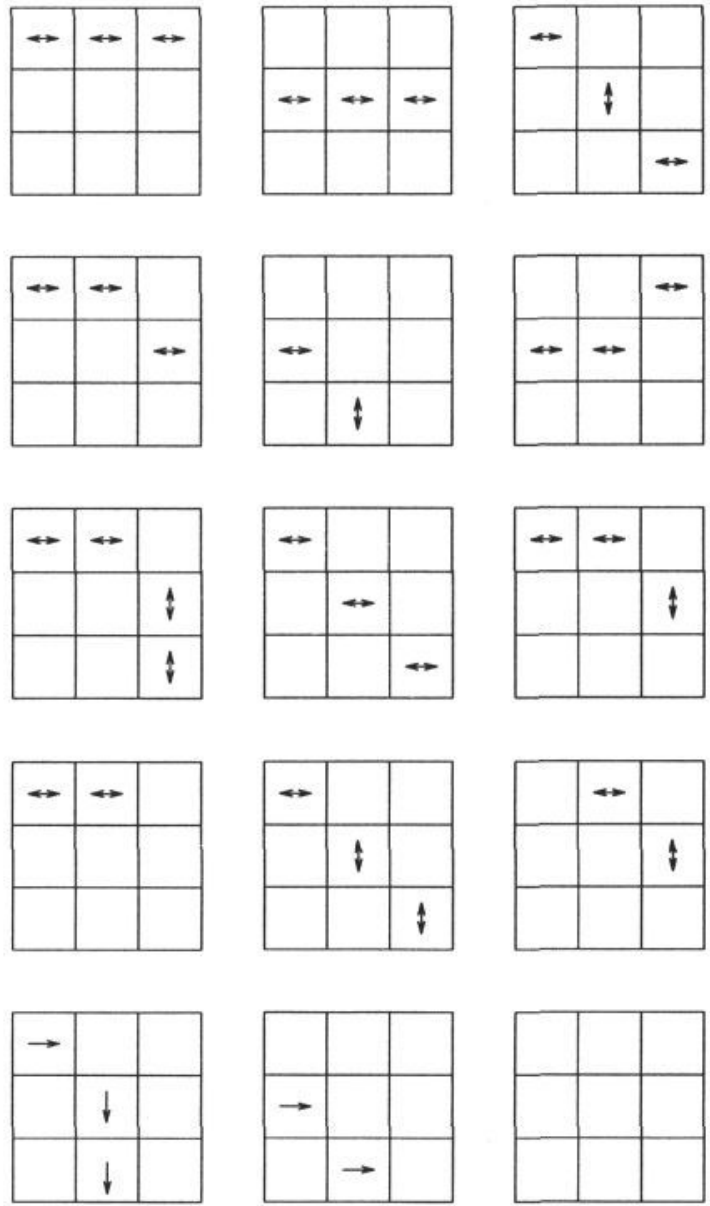

Figure 1: Contour dictionary

dictionary ingredient, the support function for multiframe feature tracking becomes

$$
\begin{aligned}
Q^{(n)}\left(\theta_{i, j}^{L}\right)=\frac{1}{P\left(\theta_{i, j}^{L} \mid \Upsilon^{L-1}\right)} . & \\
& \sum_{\Upsilon^{L} \in \Theta}\left\{\prod_{(k, l) \in I_{i, j}^{L}-(i, j)} \frac{P^{(n)}\left(\lambda_{k, l}^{h}\right)}{P\left(\lambda_{k, l}^{h} \mid \Upsilon^{L-1}\right)}\right\} P\left(\Upsilon^{L} \mid \Upsilon^{L-1}\right)
\end{aligned}
$$

This formula differs from its single frame counterpart in two important respects. The model of label structure is expressed via interframe conditional probabilities, i.e $P\left(\Upsilon^{L} \mid \Upsilon^{L-1}\right)$, rather than via the single frame joint prior, i.e. $P\left(\Upsilon^{L}\right)$. Secondly, the initial probabilities $P^{(0)}\left(\theta_{i, j}^{L}\right)=P\left(\theta_{i, j}^{L} \mid \underline{x}_{i, j}^{L}, \Upsilon^{L-1}\right)$, depend on information derived from the previous frame. In the next Section we will describe some modelling ingredients that will allow us to construct realistic feature tracking methods based upon this novel relaxation scheme. 


\section{Modelling Feature Tracking}

Our modelling approach differs from existing alternatives $[1,7,10]$ by virtue of the way in which it simultaneously accommodates interframe contour deformations, statistical intensity variations, and, the local structure of contours. This process is conventionally approached by either the construction of elastic contour models [10] or via predictive statistical models such as Kalman filters [12]. In our approach the statistical origins of feature variability are captured at two levels. Intensity variations are modelled by the Gaussian noise model while positional variations resulting in contour deformations are captured by the distribution of inter-feature distances. Local contour structure remains a hard constraint represented by a dictionary which may be exploited to remove noise while enhancing 2D connectivity and smoothness.

\subsection{Contour Development}

In the application described here we intend concentrating our modelling of interframe feature persistence upon the development of models for the initial probabilities; the resulting distibution functions have many feature in common with local deformable template models [15]. The role of the interframe conditional priors is therefore to model the development of acceptable contour structure among the persistent features. Although there is clearly a considerable body of information to be tapped in developing effective inter-frame contour models, here we will simply assume that this is represented by a set of contours to have met certain interframe consistency requirements. Our modelling of these requirements is based on the dictionary items satisfying constraints on acceptable interframe variation in contour orientation and curvature. We therefore partition the dictionary items on frame $L$ into sets deemed acceptable according to the consistent labellings obtained on frame $L-1$; we denote this acceptable set by $S\left(\Upsilon^{L-1}\right)$. In order to compute the inter-frame conditional priors, we apportion the available probability mass uniformly among the dictionary items belonging to the partition $S\left(\Upsilon^{L-1}\right)$ i.e.,

$$
P\left(\Upsilon^{L} \mid \Upsilon^{L-1}\right)= \begin{cases}\frac{1}{\int S\left(\Upsilon^{L-1}\right) \mid} & \text { if } \Upsilon^{L} \in S\left(\Upsilon^{L-1}\right) \\ 0 & \text { otherwise }\end{cases}
$$

With the joint conditional priors to hand, the single object conditional priors $P\left(\theta_{i, j}^{L} \mid \Upsilon^{L-1}\right)$ may be computed from the relative frequencies of the various object labels in the partioned dictionary. In doing this we implicitly assume that the single object priors are independent of the bulk of contour information contained in the slice $L-1$, i.e. $P\left(\theta_{i, j}^{L} \mid \Upsilon^{L-1}\right)=P\left(\theta_{i, j}^{L-1}\right)$. This is a physically plausible assumption; it means that $a$ priori we have no prejudices about the location of feature and non-feature points, it is only when evidence is to hand that we modify our expectations.

\subsection{Interframe Feature Persistence}

The second model ingredient necessary to apply the inter-frame relaxation formula to the MRI feature tracking task is a set of initial probabilities for pixels belonging to feature and non-feature classes. In assigning these probabilities we would like to capture the acceptable deformation of feature contours between different frames. This is clearly not an easy task. It is well known that even within a single frame, the contrast of a particular feature contour will vary greatly along its physical length. Inter-frame contrast variations may be anticipated to present more severe problems. Since feature contours on individual frames are the 2D manifestations of 3D surfaces [14], they may be expected to 
display considerable positional displacement; this problem is exagerated by the non-cubic MRI data being used in our study. For these reasons, we would like to avoid a model that is based on the constancy of either intensity or local spatial proximity. Infact, if this latter requirement were the sole demand placed upon our model, then interframe persistence could be completely encapsulated by local contour consistency using the dictionary partition idea described above.

Our adopted modelling philosophy is therefore to address the issue of inter-frame persistence at the symbolic level of labelled contours and to exploit non-local information. Our basic assumption is that the labelled feature pixels in the preceding frame of a sequence represent mutually exclusive seeds from which feature points on the current frame may have developed. We may therefore compute the conditional measurement density for the tentative feature point at position $(i, j)$ in frame $F^{L}$ by summing over the contributing densities from the hard-labelled feature points in frame $F^{L-1}$, with the result

$$
\begin{aligned}
& p\left(\underline{x}_{i, j}^{L}, \Upsilon^{L-1} \mid \theta_{i, j}^{L}=\epsilon\right)= \\
& K \sum_{(k, l) \in F^{L-1}} p\left(\underline{\mathrm{x}}_{i, j}^{L}, \theta_{k, l}^{L-1}=\epsilon \mid \theta_{i, j}^{L}=\epsilon\right) P^{(\infty)}\left(\theta_{k, l}^{L-1}=\epsilon\right)
\end{aligned}
$$

where $K$ is a normalising constant and $\epsilon$ represents a combined horizontal and vertical feature label. The above expression for the conditional density effectively blurs the hardlabelled contours from frame $F^{L-1}$ to produce an evidential field for potential contours in frame $F^{L}$. The hard label information is represented by $P^{(\infty)}\left(\theta_{k, l}^{L}=\epsilon\right)$ which indicates whether or not the pixel $(k, l)$ is assigned to the feature class in frame $L-1$. Evidential information is represented by the individual densities $p\left(\underline{x}_{i, j}^{L}, \theta_{i, j}^{L-1}=\epsilon \mid \theta_{k, l}^{L}=\epsilon\right)$ for the features contained within frame $F^{L}$ to have originated from contours in frame $F^{L-1}$. It is these densities that form the critical and novel ingredient of our contour tracking method. They model the development of tentative feature points on the frame $F^{L}$ from the labelled feature points on the frame $F^{L-1}$. Their role is to capture the spatial variations in contour position on different frames; they account for interframe deformation of $2 \mathrm{D}$ contours originating from the surface geometry of 3D structures.

In developing our model of the underlying variations in contour position due to surface geometry, we make only one simple and relatively unrestrictive assumption. We assume that each feature point in the frame $F^{L-1}$ is associated with a local plane and that feature points in frame $F^{L}$ associate to these planes under a uniform distribution of orientation. If the two frames are separated by a physical distance $t$, then provided that the surface normals of our implicit or virtual planes are uniformly distributed on the unit sphere, then the required probability density is specified in terms of the distance between pairs of feature points as measured on the frames

$$
p\left(\underline{\mathbf{x}}_{i, j}^{L}, \theta_{k, l}^{L-1}=\epsilon \mid \theta_{i, j}^{L}=\epsilon\right)=\frac{t}{\pi\left[t^{2}+(i-k)^{2}+(j-l)^{2}\right]}
$$

This probability density is unimodal and is close in form to the Gaussian. Moreover, the density expansion appearing in equation (4) has many features in common with the generalised deformable models of Yuille [15]. Our approach to the modelling of interframe contour variation may therefore be thought of as being close in spirit to a variety of elastic [1] or deformable $[7,10]$ template methods which have proved popular in tracking 


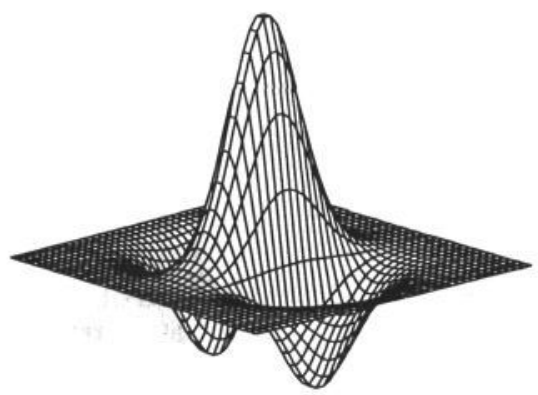

Figure 2: Line Detection Kernel

problems. In fact, Zucker et al [16] have already pointed out that contour refinement by relaxation operations can be regarded as a local form of snake dynamics [10]. The novelty of our approach stems from its implicit surface modelling to compute a distribution of acceptable interframe contour movements. In the next section we will demonstrate how this distribution may be combined with intensity information for refinement by relaxation operations. It is this combination process that couples interframe contour movement to intensity features in the individual frames.

\subsection{Feature Probabilities}

In the experimental section of this paper, we will be interested in tracking the cranial outline in an MRI image stack. This structure presents itself as an intensity extremum in the individual frames. Such ridge feature are best characterised by directional Mexican hat filters of the type shown in Figure 2; examples include the second-derivative of Gaussian and Gabor filters [9]. We have recently described a statistical framework which may be used to produce a probabilistic representation of the output of an orientational linedetection filter bank [9]. Details of this method are outside the scope of this paper. Suffice to say that the basic modelling method is to compute the probability density function for the combined filter bank for uniform image regions under the action of additive Gaussian noise. If $\underline{x}_{i, j}^{L}$ represents the response of a pair of vertical and horizontal ridge-filters at the pixel $(i, j)$, then the conditional measurement density for pixels belonging to the non-feature class $\phi$ is

$$
p\left(\underline{\mathbf{x}}_{i, j}^{L}, \Upsilon^{L-1} \mid \theta_{i, j}^{L}=\phi\right)=\frac{1}{2 \pi} \frac{1}{\sqrt{|\Sigma|}} \exp \left[-\frac{1}{2}\left(\underline{\mathbf{x}}_{i, j}^{L}\right)^{T} \Sigma^{-1}\left(\underline{\mathbf{x}}_{i, j}^{L}\right)\right]
$$

In the above expression $\Sigma$ represents the variance covariance matrix for the different components of the filter stack. The elements of this matrix may be computed from the known autocorrelations and cross-correlations of the filters used to determine the orientational feature responses; its only parameter is the variance of the additive image noise. It is worth stressing that we adopt very different philosophies in modelling the feature-class and non-feature class densities In the feature case we have assumed that the density depends only upon the geometry of the feature points, while in the non-feature case the density is determined purely by the filter responses. The motivation underpinning this approach is that although the intensity appearance of noisy 3D features will vary 


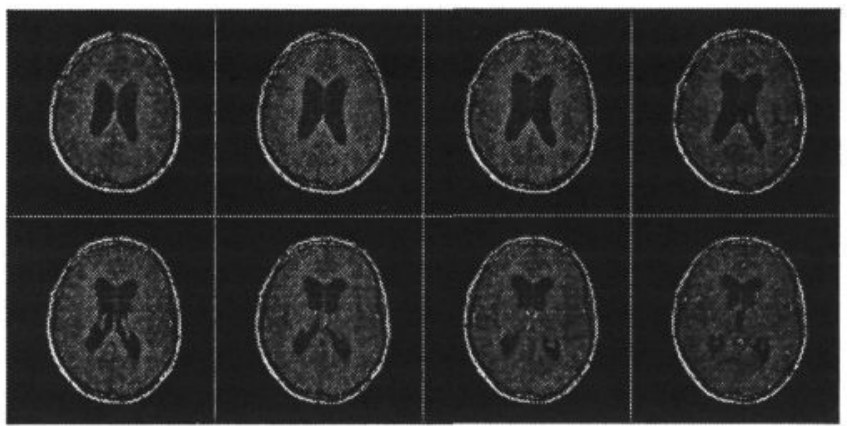

Figure 3: MRI Slices

greatly from frame to frame, their geometry is more stable and may be exploited to much greater effect in modelling interframe persistence. However, in establishing tentative feature positions on individual frames, there is clearly no alternative to using intensity information.

We are now in a position to compute the initial probabilities required to seed the relaxation process. With the conditional measurement densities for the feature and nonfeature classes to hand, this may be effected by a simple application of the Bayes formula. For instance for the feature-class

$$
\begin{aligned}
P\left(\theta_{i, j}^{L}=\right. & \left.\epsilon \mid \underline{\mathrm{x}}_{i, j}^{L}, \mathrm{\Upsilon}^{L-1}\right)= \\
& \frac{p\left(\underline{\mathrm{x}}_{i, j}^{L}, \Upsilon^{L-1} \mid \theta_{i, j}^{L}=\epsilon\right) P\left(\theta_{i, j}^{L}=\epsilon\right)}{p\left(\underline{\mathrm{x}}_{i, j}^{L}, \Upsilon^{L-1} \mid \theta_{i, j}^{L}=\epsilon\right) P\left(\theta_{i, j}^{L}=\epsilon\right)+p\left(\underline{\mathrm{x}}_{i, j}^{L}, \Upsilon^{L-1} \mid \theta_{i, j}^{L}=\phi\right) P\left(\theta_{i, j}^{L}=\phi\right)}
\end{aligned}
$$

The null-feature class probabilities may be obtained in an analogous way. The densities required are specified by equations (4), (5) and (6). The resulting distribution of initial probabilities effectively couples feature characteristics from filtering with information derived from hard-labeled contours; it can be regarded as a providing a combined index for the existence of features on succeeding frames. It is the application of the relaxation operator defined by equations(2) and (3) that imposes contour consistency on the distribution of probabilities through the use of inter-frame persistence constraints contained within the dictionary.

\section{Experiments}

In order to demonstrate the effectiveness of our feature tracking method, we have taken the application of detecting the cranial outline in an MRI stack consisting of 36 frames. Figure 3 shows 8 consecutive frames from the data-set used for this study. The desired features exhibit variations in contrast, position and width. Figure 4 contains the result of applying our inter-frame tracking method. For comparison Figure 5 shows the result of applying independent single frame feature detection to the same data. There is a marked difference in the two sets of results. The single frame contours are subject to noise and display locally erratic changes in direction. By contrast, the multi-frame method produces contours that are noise free and smooth. 


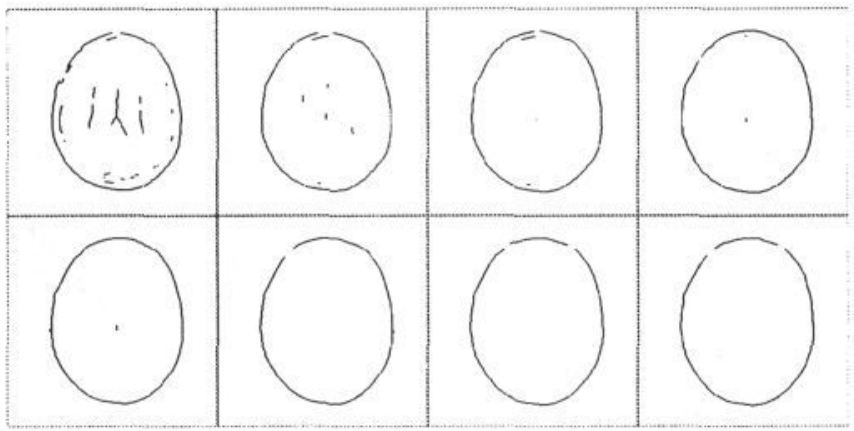

Figure 4: Results of multiframe relaxation

The tracking version of the relaxation algorithm is initialised on a representative contour. Although there are genuine intermitent gaps, the subsequent contours quickly recover their overall integrity. The overall movement of the contours accounts for tens of pixels over the sequence. Despite this fact the cranial outline is maintained; there is little evidence for loss of contact during the tracking operation. Transient clutter although manifest in the single level results, is notably absent from the multiframe output. Infact, much of the clutter in the single level approach is associated with contamination by edge and other non-ridge intensity artifacts [9]. The ability of the feature tracker to reject transient features appears to enhance its capacity to cleanly locate pure ridge structure.

\section{Conclusions}

We have described a novel feature tracking method. It is based on an interframe relaxation technique. This method draws upon both intra-frame and inter-frame constraints regarding the behaviour of acceptable contour structure. Intra-frame information is represented in the form of a dictionary of local contour structure combined with a statistical model of the response of a set of directional feature detection operators. The inter-frame ingredient represents the novel model component described in this paper; it is encapsulated by an implicit model of the underlying surface structure of 3D feature points. The model is represented in terms of a series of unimodal probability densities whose single parameter is the inter-frame distance.

We have experimented with the resulting feature detection method on cranial MRI data. Here the method significantly outperforms its single frame counterpart in terms of its ability to extract noise-free and smooth feature contours.

\section{References}

[1] Blake A., Curwen, R. and Zisserman A., "Affine Invariant Contour Tracking with Automatic control of Spatiotemporal Scale",Proceedings of the Fourth International Conference on Computer Vision, pp 66-75, 1993.

[2] Canny J.F., “A computational approach to edge detection”, IEEE PAMI, PAMI 8, pp. 679-698, 1986.

[3] Deriche R., "Optimal edge detection using recursive filtering", International Journal of Computer Vision', 2, pp.167-187, 1987.

[4] Flynn P.J., and Jain A.K., "On reliable curvature estimation", IEEE Computer Vision and Pattern Recognition Conference, pp. 110-116, 1989. 


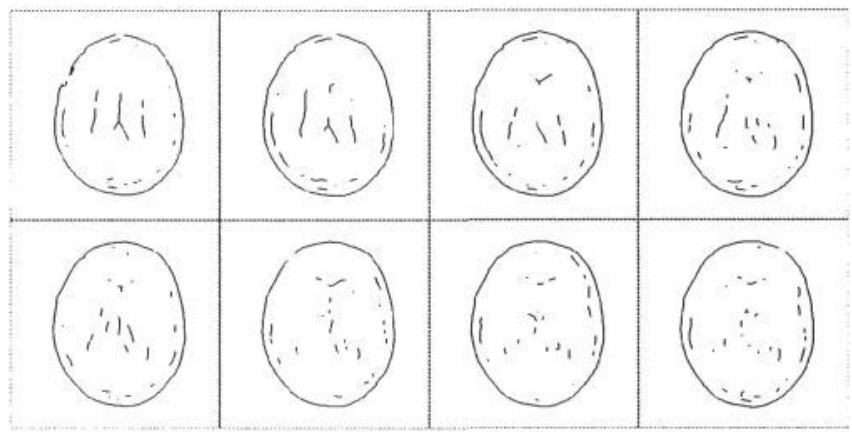

Figure 5: Results of single frame relaxation

[5] Hancock E.R. and Kittler J., "Edge labelling using dictionary based relaxation", IEEE PAMI, PAMI 12, pp.165-181, 1990.

[6] Hancock E.R. and Kittler J., "Adaptive estimation of hysteresis thresholds", Proceedings IEEE Computer Vision and Pattern Recognition Conference,pp. 196-201, 1991.

[7] Gueziec A., "Large Deformable Splines, Crest Lines and Matching", Proceedings of the Fourth International Conference on Computer Vision, pp 650-657, 1993.

[8] Hancock E.R. and Kittler J., "Multiresolution edge labelling using hierarchical relaxation", $11^{\text {th }}$ International Conference on Pattern Recognition, Volume 2, pp.140-144, 1992.

[9] Hancock E.R., "Resolving Edge-Line Ambiguities using Probabilistic Relaxation", Proceedings IEEE CVPR Conference, pp300-306, 1993.

[10] Kass M, Witken A., and Terzopoulos M., "Constraints on deformable models: Recovering 3D Shape from non-rigid motion”, Artif. Intell.,36, pp 91-123, 1988

[11] Monga O., Deriche R., and Malandain G., "Recursive Filtering and edge closing; two primary tools for 3D edge detection”, Image and Vision Computing, 9, pp. , 1991.

[12] Monga O., Ayache N. and Sander P., "Modelling uncertainty for estimating local surface geometry", Proceedings of the $7^{\text {th }}$ Scandinavian Conference on Image Analysis, Volume 1, pp. 403-410, 1991.

[13] Sander P.T. and Zucker S.W., "Singularities of principal direction fields from 3D images", IEEE PAMI, PAMI 14, pp. 309-317, 1992.

[14] Sander P.T. and Zucker S.W., "Inferring surface trace and differential structure from 3D images”, IEEE PAMI, PAMI 12, pp.833-854, 1990.

[15] Yuille A., "Generalised Deformable Models, Statistical Physics and Matching Problems", Neural Computation, 2 , pp. 1-24, 1990.

[16] Zucker S., David C., Dobbins A., and Iverson L., "The Organisation of Curve Detection: Coarse Tangent Fields and Fine Spline Coverings", Proceedings of the Second International Conference on Computer Vision, pp. 586-577, 1988. 


$$
\text { . }
$$

